\title{
Hypothesis: hair cover can protect against invasive melanoma on the head and neck (Australia)
}

\author{
Adèle C. Green • Michael Kimlin • Victor Siskind • \\ David C. Whiteman
}

Received: 2 May 2006/ Accepted: 13 July 2006

(C) Springer Science+Business Media B.V. 2006

\begin{abstract}
The anatomic distribution of cutaneous melanoma reflects people's levels and patterns of sun exposure. While examining trends of incident invasive melanomas by site in recent decades in Australia we noted significant increases in incidence on the ears but not the face or any other site in women younger than 40 years, by $6 \%$ (95\% confidence interval [CI] $2-10 \%$ ) per year, and $40-59$ years by $7 \%$ (95\% CI $4-10 \%)$ per year. Men of the same age showed no corresponding changes in ear melanoma. However incidence rates of ear melanoma in general were fourfold higher in males than females in Australia. Further, using data from the National Cancer Institute's Surveillance, Epidemiology and End Results (SEER) Program, rates of invasive melanoma on the ear were found to be sevenfold higher in males than females in the US population in the same period. Higher rates of scalp and neck melanomas were also seen in men and women in both populations. We therefore speculated that the isolated rises of ear melanoma in younger women in Australia, and the higher overall rates of ear, scalp and neck melanoma in men compared with women, reflect differences in hair coverage. We tested the specific hypothesis that hair cover reduces sun exposure of the ears using experimental manikins and found that hair cover of the ear reduced solar ultraviolet-B exposure
\end{abstract}

A. C. Green $(\bowtie) \cdot$ V. Siskind · D. C. Whiteman Queensland Institute of Medical Research, PO Royal Brisbane Hospital, Brisbane, Queensland 4029, Australia e-mail: adele.green@qimr.edu.au

M. Kimlin

School of Public Health, Queensland University of Technology, Brisbane, Australia by $81 \%[\mathrm{SE} \pm 8]$ compared with uncovered ears. We conclude that hair cover can protect against invasive melanoma on the ear and may similarly protect on the scalp and neck. When discretionary, hair may be an important additional factor to be considered for melanoma prevention.

Keywords Melanoma $\cdot$ Ear $\cdot$ Solar ultraviolet radiation $\cdot$ Protection $\cdot$ Hair

\section{Introduction}

Sun exposure is the major environmental cause of melanoma [1] and so the anatomic distribution of melanoma in Caucasian populations largely reflects relative levels of sun exposure of different body sites [2]. Similarly in a given population, changes in site distribution of melanoma have long been known to offer clues about change in people's sun-related behavior [3]. For example Magnus [4] analyzed melanoma incidence rates by age cohort and tumor site in Norway 1955-1977 and found that trunk and lower limb melanomas had increased much more than face and neck melanomas among younger generations, consistent with changes in clothing and sun-tanning habits in the first half of the twentieth century. Here we describe an isolated rise in the incidence of melanoma of the ear in women under 60 years of age in Australia. This distinct increase of invasive melanoma on a discrete site indicated some diminution in covering of the ear in young women in preceding decades. While a change in hat wear was one possible explanation, we speculated that a change in hair cover may also explain the observed rise, and that less hair coverage of the 
head among men in general may explain their higher relative incidence of melanoma on the ears and scalp compared with women [2]. When we searched for published data regarding the sun protection factor of hair we found none. Hence we set out to test the specific hypothesis that hair cover protects the ear from sun exposure using experimental manikins.

\section{Recent trends in melanoma in Australia}

Based on data for 109,062 invasive melanomas assembled by the Australian Institute of Health and Welfare from cancer registries in all Australian states and territories, trends in incidence of cutaneous melanoma in Australia 1982-1999 by sex and site were examined. Sites of invasive melanoma were pre-classified by cancer registries according to a standard protocol. For each site annual notifications were provided with age in 5year bands. Population estimates by age and sex were obtained for each year from the Australian Bureau of Statistics. Annual sex-specific incidence rates of melanoma age-standardized to the WHO World Population were computed for each site and relative rates of increase were calculated for three age bands, less than 40 years, 40-59 years and 60 years and over, by regressing the logarithms of these rates on years.

The average relative change in melanoma incidence rates over all sites each year was estimated as 3.2\% (99\% CI $3.0-3.4 \%)$ in males and $1.5 \%$ (99\% CI $1.3-1.8 \%)$ in females but increases varied by sex and age. Highest increases were seen in men 60 years and over $(4.4 \%$ per year, $95 \%$ CI $4.2-4.6 \%$ ), followed by women 60 and over and men $40-59$ years ( $2.9 \%$ per year), and then by women aged $40-59$ years (1.5\% per year) (Table 1$)$. In contrast persons under 40 years showed little to no relative increase in invasive melanoma in the last two decades: young men showed a $1.4 \%$ annual increase of borderline significance and women under 40 years showed virtually no change in melanoma incidence (Table 1).

\section{Recent site-specific trends in melanoma on the head and neck in Australia}

Site-specific trends in melanoma for males and females within each age group reflected the above trends for all sites combined, such that men 60 and over generally had the highest relative increases on each anatomic site and women under 40, the least (data not shown for trunk and limbs). The striking exception was a significant $6.1 \%$ increase $(95 \%$ CI $2.4-9.9 \%)$ in incidence rates of melanoma on the ears in women under 40 years based on 106 invasive tumors diagnosed in the study period. A similar rise of 7\% (95\% CI 3.6-10.4\%) was seen for ear melanomas in women 40-59 years based on 130 invasive tumors (Table 1). The incidence rates of melanoma on the face in young women and those under $40-59$ years showed little change $(0.9 \%$ and $0.0 \%$ respectively), and similarly on the scalp and neck (data not shown).

In contrast, young men showed no significant relative increases of melanoma on the ear or any other sites on the head and neck in the same period. Men aged 4059 years also showed no significant relative increases of melanoma on the ear but modest relative increases in melanoma on the face of around $3 \%$ per year (Table 1 ) and also on the scalp and neck.

\section{Sex-specific differences in incidence rates of melanoma on the head and neck}

Between 1982 and 1999 in Australia the overall agestandardized incidence rate of ear melanoma in males was 0.91 (99\% CI 0.85-0.97) per 100,000, 4 times higher than the corresponding rate of 0.24 (99\% CI $0.21-0.26)$ per 100,000 in females. The male excess was seen in all age groups though the difference in rates of ear melanoma between young men and young women was small by the end of the study period, compared with the sevenfold sex-specific difference seen in older men and women over 60 . The incidence rate of scalp and neck melanoma (sites pre-combined) in males was 2 times higher than in females $(2.18,99 \%$ CI $2.09-2.27$ and 1.08 , 99\% CI 1.02-1.14 per 100,000 respectively) though again the differences were narrowed in the youngest age group. Incidence rates of melanoma on the face were slightly higher overall in males than females $(2.62,99 \%$ CI $2.52-$ 2.72 and 2.06 , 99\% CI $1.98-2.14$ per 100,000 respectively) but were no different in men and women under 40 years (Table 1 ).

Differences in incidence rates of melanoma on the ear, scalp, face and neck were seen even more clearly in an earlier unrelated study of the Queensland data for 1987 [2] where site-specific incidence rates of melanoma had been calculated and further adjusted for relative body surface area. Incidence of invasive melanoma on the ear in Queensland males was 209 per unit area of skin per 100,000 per year compared with 50 per unit area of skin per 100,000 per year in females, again a fourfold difference. On the scalp there was a 20 -fold greater incidence in males than females and on the neck a fourfold difference. Again rates on the face in males and females showed very little difference in Queensland in 1987 [2]. 
Table 1 Age-standardized incidence of melanoma on head and neck sites and all sites in Australia 1982-1986 and $1995-1999$ by age and sex

\begin{tabular}{|c|c|c|c|c|c|}
\hline \multirow[t]{2}{*}{ Site } & \multirow[t]{2}{*}{$\begin{array}{l}\text { Age } \\
\text { (years) }\end{array}$} & \multirow[t]{2}{*}{$\begin{array}{l}\text { No. of } \\
\text { melanomas }\end{array}$} & \multicolumn{2}{|c|}{$\begin{array}{l}\text { Incidence rates per } \\
100,000\end{array}$} & \multirow[t]{2}{*}{$\begin{array}{l}\% \text { Annual relative increase } \\
(95 \% \mathrm{CI})\end{array}$} \\
\hline & & & 1982-1986 & 1995-1999 & \\
\hline \multicolumn{6}{|l|}{ Males } \\
\hline \multirow[t]{3}{*}{ Ear } & $<40$ & 179 & 0.15 & 0.22 & $2.0(-0.9-5.0)$ \\
\hline & $40-59$ & 426 & 0.95 & 1.31 & $1.9(0.0-3.8)$ \\
\hline & $\geq 60$ & 1,115 & 3.29 & 6.46 & $4.2(3.0-5.4)$ \\
\hline \multirow[t]{3}{*}{ Face } & $<40$ & 392 & 0.34 & 0.50 & $2.6(-0.7-4.5)$ \\
\hline & $40-59$ & 1,125 & 2.48 & 3.85 & $3.2(2.1-4.4)$ \\
\hline & $\geq 60$ & 3,546 & 11.06 & 20.30 & $3.7(3.0-4.4)$ \\
\hline \multirow[t]{3}{*}{ All sites } & $<40$ & 10,731 & 8.91 & 11.98 & $1.4(1.0-1.8)$ \\
\hline & $40-59$ & 19,898 & 43.38 & 64.65 & $2.8(2.5-3.1)$ \\
\hline & $\geq 60$ & 27,957 & 85.34 & 166.53 & $4.4(4.2-4.6)$ \\
\hline \multicolumn{6}{|l|}{ Females } \\
\hline \multirow[t]{3}{*}{ Ear } & $<40$ & 106 & 0.08 & 0.17 & $6.1(2.4-9.9)$ \\
\hline & $40-59$ & 130 & 0.23 & 0.53 & $7.0(3.6-10.4)$ \\
\hline & $\geq 60$ & 226 & 0.58 & 0.92 & $1.9(-0.8-4.6)$ \\
\hline \multirow[t]{3}{*}{ Face } & $<40$ & 451 & 0.40 & 0.54 & $0.9(-0.9-0.7)$ \\
\hline & $40-59$ & 840 & 2.48 & 2.34 & $0.0(-1.3-1.4)$ \\
\hline & $\geq 60$ & 3,508 & 9.73 & 15.28 & $2.4(1.8-3.1)$ \\
\hline \multirow[t]{3}{*}{ All sites } & $<40$ & 13,494 & 13.49 & 15.29 & $0.3(0.1-0.7)$ \\
\hline & $40-59$ & 17,328 & 44.24 & 54.69 & $1.5(1.2-1.8)$ \\
\hline & $\geq 60$ & 19,654 & 55.74 & 87.05 & $2.9(2.6-3.2)$ \\
\hline
\end{tabular}

To assess whether comparable patterns were seen in other populations, age-standardized incidence rates of melanoma per 100,000 for 1982-1999 were calculated for sub-sites of the head and neck for the regions covered by the National Cancer Institute's Surveillance, Epidemiology and End Results (SEER) Program of population-based cancer registries [5]. In males compared with females there was a sevenfold higher incidence rate of ear melanoma and a threefold higher rate of scalp and neck melanoma compared with a less than twofold higher incidence rate of melanoma on the face.

\section{Hypothesis}

The systematically higher incidence of invasive melanoma on the ear, scalp and neck compared with the face in males and females is consistent with the general hypothesis that hair cover can offer long-term protection of the skin from sun exposure. We tested the specific hypothesis that hair cover of the ear protects it from solar ultraviolet (UV) radiation by conducting an experiment using manikin head-forms in which the difference in solar UV radiation to ears with and without hair cover was measured. We used four manikin head-forms: three wearing human hairpieces (blonde, brown and black) covering the ears and one uncovered (Fig. 1). These were set facing north on a
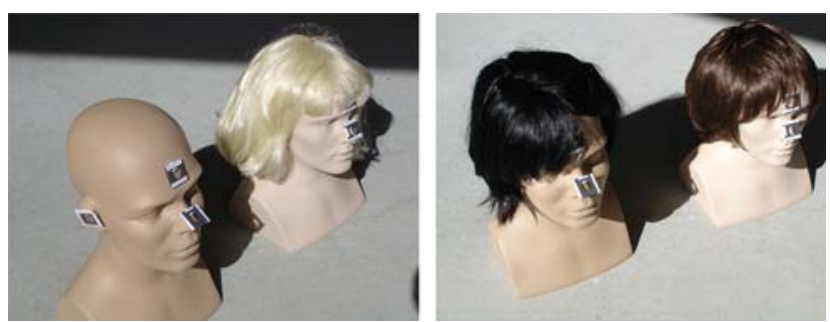

Fig. 1 Measurement of solar ultraviolet radiation on ears in relation to hair cover using head-forms and polysulfone dosimeters

concrete floor and exposed to natural sunlight in Brisbane $\left(27^{\circ} \mathrm{S}\right)$ from 11 am to $1 \mathrm{pm}$ Australian Eastern Standard Time daily for five consecutive days in May 2005. Cloud cover was zero and surface reflectivity less than $7 \%$. Sun exposure was measured by UV-sensitive polysulfone dosimeters placed on the ears and nose of each manikin.

Ears covered by hair received on average $81 \%$ [SE \pm 8 ] less solar UV radiation than bare ears when exposed to the sun. As a control site on the face, the corresponding solar UV exposures to the noses of the manikins were monitored (Fig. 1) and showed a slight variation during the experiment in the reverse direction, namely a $5 \%[\mathrm{SE} \pm 3.4]$ increase in UV radiation to the wigged manikins compared with the bare manikin. 


\section{Discussion}

These experimental data support our hypothesis that hair cover offers substantial sun protection of the skin of the ear and by extrapolation, the skin of the scalp and back of the neck as well if hair is worn long. Taken together with the recently observed change in incidence of invasive melanoma on the ear in women in Australia and the consistent pattern in Australia and the United States of lower incidence of melanoma in females on the scalp, ear and neck - sites that are more frequently covered or screened by hair than in males, the evidence suggests that hair cover can indeed protect against invasive melanoma on these sites.

Other explanations for the observed site- and sexspecific patterns can be considered. Change in diagnostic trends was unlikely to explain the observed rise in ear melanomas in younger women since in situ melanomas were not included in the analyses. One possibility is that the distinct increase of ear melanomas in Australian women under 60 was due to the loss of some other form of physical protection. For example the rise in popularity of caps in the 1980s resulted in a decrease in cover of the ear but not the face in young women who wore brimmed hats in preceding decades [6]. If this were the sole explanation, some analogous rise in the rate of ear melanomas in men might have been expected as an aftermath as well, but has been observed neither in Australia nor the US. Another cause of increasing sun exposure of the ears in young women may have been the upsurge in women's participation in outdoor sport that also occurred in the 1970s and 1980s [7] if caps rather than hats were worn. However even if change from hats to caps did explain the recent rise in incidence of ear melanomas in Australian women, difference in hat wear between the sexes does not explain the systematically higher rates of invasive melanoma in men than in women across the various sub-sites of the head and neck with less discrepant rates on the face. (Indeed facial hair cover in men with beards may mitigate the effect of overall higher outdoor exposure of men than women for facial melanomas). We also speculated that younger women's hair styles may have changed over time and become shorter leading to greater exposure of the ears and neck, but there is little evidence available to address this question.

Finally in view of the known sex-specific differences in melanoma incidence on sites such as the scalp, it is surprising that the sun-protection factor of human hair has never been measured before. This absence of quantitative data contrasts with the extensive information available about the sun-protection provided by clothing and sunscreens $[8,9]$. If our general hypothesis is confirmed, hair cover of the ears in particular would add another potential means of melanoma prevention, especially in high-risk populations, among individuals in whom amount of hair cover is discretionary.

Acknowledgments Thanks to Dr Penny McBride for very helpful suggestions on the manuscript. Michael Kimlin is funded through a Queensland Government "Smart State" Fellowship, and David Whiteman is a Senior Research Fellow of the National Health and Medical Research Council of Australia.

\section{References}

1. International Agency for Research on Cancer (1992) IARC monographs on the evaluation of carcinogenic risks to humans. Vol. 55, Solar and ultraviolet radiation. IARC, Lyon

2. Green A, MacLennan R, Youl P, Martin N (1993) Site distribution of cutaneous melanoma in Queensland. Int J Cancer 53:232-236

3. Bulliard JL, Cox B (2000) Cutaneous malignant melanoma in New Zealand: trends by anatomical site, 1969-1993. Int J Epidemiol 29:416-423

4. Magnus K (1981) Habits of sun exposure and risk of malignant melanoma. Cancer 48:2329-2325

5. Ries LAG, Eisner MP, Kosary CL, et al (eds) (2003) SEER cancer statistics review, 1975-2000. National Cancer Institute, Bethesda MD. http://seer.cancer.gov/csr/1975_2000

6. The Baseball Cap. http://www.villagehatshop.com/baseball_caps.html. Accessed 28 March 2006

7. History of Women in Sport in Australia. Available at: http:// www.ausport.gov.au/fulltext/2001/ascpub/women_history.asp. Accessed 28 March 2006

8. Laperre J, Gambichler T (2003) Sun protection offered by fabrics: on the relation between effective doses based on different action spectra. Photodermatol Photoimmunol Photomed 19:11-16

9. Herzog B, Mongiat S, Quass K, Deshayes C (2004) Prediction of sun protection factors and UVA parameters of sunscreens by using a calibrated step film model. J Pharm Sci 93:17801795 